\title{
Incidental findings in computed tomography scans: Concern for clinicians
}

\author{
Sharan Badiger ${ }^{*}$ and Prema Akkasaligar ${ }^{2}$ \\ ${ }^{1}$ Department of Medicine, Sri B M Patil Medical College, Vijayapur, Karnataka, India \\ ${ }^{2}$ Department of Computer Science, Dr. P G Halakatti College of Engineering and Technology, Vijayapur, Karnataka, India
}

\begin{abstract}
The importance of computed tomography scans in patients with suspected trauma was to assess the damage of organs or structures as a result of trauma; however, sometimes findings unrelated to the trauma and not concerned to the immediate patient care are detected. These findings are called as "incidental findings" in trauma patients. Proper clinical examination and focused management follow up of incidental findings can result in appropriate patient care and prognosis.
\end{abstract}

\section{Introduction}

Modern medical science has seen tremendous growth in the use of imaging technology. The objective of imaging application is to use it as a diagnosing tool in helping medical practitioners and play an important role in the management of patients. The quality of medical imaging and access to new devices has increased, assuming that 'newer is better' [1]. The number of incidental findings has increased due to growing number of imaging techniques. The pulmonary nodules on chest and 'incidentalomas' on abdominal imaging are classical examples for the same [2,3]. The incidental findings help in additional medical care, diagnostic test, procedure and treatment to the patients is known as cascade effect [4]. Clinicians should know how to treat the incidental findings on imaging, in-order to avoid any fatal consequences. The absence of evidence-based control studies has led to unawareness of treatment and prognostic significance and implications for incidental finding. The objective of this review was to determine the incidence, clinical significance and frequency of incidental findings on computed tomography $(\mathrm{CT})$ in trauma patients.

Medical imaging technology has experienced a dramatic change in the last few years. Medical imaging refers to the techniques and processes used to create images of the human body for various clinical purposes such as medical procedures and diagnosis or medical science including the study of normal anatomy and function. With the growth of computers and image technology, medical imaging has greatly influenced the medical field. The diagnosis of a health problem is now highly dependent on the quality and the credibility of the image analysis. This paper deals with the various aspects and types of medical imaging [5].

Image denoising is a procedure in digital image processing aiming at the removal of noise, which may corrupt an image during its acquisition or transmission, while retaining its quality. Image denoising still remains the challenge for researchers because noise removal introduces artefacts and causes blurring of the images [6].

The clinician relies heavily on techniques in diagnosing pericardial disease that demonstrate the presence of pericardial effusion. Currently available investigative tools that aid in the detection of pericardial effusion include electrocardiography, echocardiography, chest X-ray, cardiac fluoroscopy, computerized tomography, magnetic resonance imaging, radionuclide scanning and pericardiocentesis [7].

Today, echocardiography is considered the procedure of choice for evaluating patients suspected of having pericardial effusion. This high place afforded to echocardiography is due to its being an accurate, easy to perform and non-invasive method. It must be remembered, however that the results obtained are entirely dependent on the knowledge, experience and technical skill of the examiner. The echocardiographer must be familiar with the ultrasonic cardiac anatomy and various intracardiac landmarks. Many previously published false-positive and false-negative echocardiograms in pericardial effusion can be directly attributed to faulty techniques [8].

Medical images are usually corrupted by noise during their acquisition and transmission. The main objective of image denoising techniques is to remove such noises while retaining as much as possible the important image features [9].

\section{Review of literature}

The incidental findings on CT after trauma evaluation are a growing concern for clinicians in regard to the diagnosis and management of those findings. It was found that 5,831 (52\%) had CT scan and 89 (1.5\%) had at least one incidental finding. The incidental findings were commonly found in abdominal-pelvis CTs. Results showed that incidental findings were higher in men and older patients. Shella Farooki, lead author of Columbus Radiology Corp. at Grant Medical Center in Columbus, $\mathrm{OH}$, in this study found that patients who were older and had a higher injury severity score were more likely to have incidental findings [10]. A majority of patients come to the emergency

*Correspondence to: Sharan Badiger, Department of Medicine, Sri B M Patil Medical College, Vijayapur, Karnataka, India, Tel: 919448434927; E-mail: sharanrb@rediffmail.com

Key words: computed tomography, incidental findings, trauma

Received: July 15, 2019; Accepted: July 30, 2019; Published: August 07, 2019 
department (ED) as trauma patients but are having the diagnosis that is not related to trauma. As a clinician, we have to pay attention to detailed clinical examination, investigations, and follow-up is important. CT scan is commonly used diagnostic screening tools in the emergency department (ED) [11]. The CT scan findings unrelated to the trauma and not related to the immediate patient care in the emergency department are defined as 'incidental findings.' The increased availability and the advances in the technology of CT scanners help in detecting incidental finding. Majority of the incidental findings are benign and require no follow-up, others require serial imaging and close supervision of the patient by their primary care clinician [12].

Earlier studies have found the rate of incidental findings in trauma patients require serial and large amount of CT scan in their study group [13-16]. The rate of incidental findings the present study is $1.5 \%$ and is lower than that in other studies with reported rate from $34-43 \%$ on abdominal CT scans [13-15]. The incidence of proper documentation and referral for follow-up of incidental findings in trauma patients ranged from $21-27 \%$. This study has explored incidental finding rates in trauma patients who underwent CT scans in the ED for trauma and subsequently referred for appropriately follow-up [15].

In another study of cranial CT scan of 2,000 cases of head trauma (HT). A normal CT scan was seen in 60.75\% (1215) and an abnormal CT scan in $39.25 \%$ (785) of patients. Out of 785 abnormal CT scan, 518 (65.9\%) lesions were related to HT. The most common CT scan HT related findings were: soft tissue swelling (8.9\%), skull fractures (4.3\%), intracranial and sub-galeal hematomas (3.4\% and $2.4 \%$ ), brain swelling (2\%) and brain contusion (1.2\%). Out of 785 abnormal CT scans, 267 (34.1\%) lesions were not related to head trauma. Incidental findings were brain atrophy (5.9\%), one calcification (5.2\%) several calcifications (2.4\%), ischemic infarct (1.9\%), and leukoaraiosis (1.3\%) [17].

In a study of 3,000 CT scans of brain of trauma patients for incidental findings, the most common incidental findings were large cisterna magna $\left(>10 \mathrm{~cm}^{3}\right)$ in 11 cases, 8 cases of tumour (3 meningioma, 2 cranio-pharyngioma, 1 oligo-dendroglioma, 1 lowgrade astrocytoma, and 1 medulloblastoma), arachnoid cyst in 7 cases, suspected lipoma, osteoma, abnormal calcifications, and hydrocephaly in 3 cases each [18].

In a data of $732 \mathrm{CT}$ scans of head trauma patients, 500 (68.3\%) were male and $232(31.7 \%)$ were female. The mean age was $27.4 \pm 19.2$. Incidental findings were detected in 22 patients $(3.1 \%)$, of them 10 (45\%) were male and $12(55 \%)$ were female patients $(P=0.019)$. The mean age of cases with incidental findings were $37.2 \pm 20.6$ years and in cases without incidental findings were $27.1 \pm 19.1$ years $(P=0.011)$. Among these, there were 5 tumors (0.7\%), 8 arachnoids cysts (1.1\%), and 5 bony lesions $(0.7 \%)$ and in 4 cases large cisterna magna (> 10 $\mathrm{cm} 3$ ) was seen [19].

\section{Conclusion}

The evaluation of trauma continues to evolve as new technology and improvise standards of patients care in ED. The CT scan has become a standard diagnostic tool in trauma patients in identifying acute osseous injuries. A higher incidence of incidental findings is found with abdominal and pelvic CT compared to spinal CT. The frequency of incidental findings is higher among men and older patients. Proper clinical examination and focused management follow up of incidental findings can result in appropriate patient care and prognosis. The diagnostic power is associated with radiation hazard.
CT- related radiation leads to increase in cancer mortality. Reduction in the scan length results in linear reduction in radiation hazards.

Incidental findings vary from minor trivial lesions to major fatal catastrophic pathologic lesions. They have to be diagnosed in patients evaluated for trauma in the widespread use of CT scan. Many of these findings require early management or referral to specialty physicians. Incidental findings in these patients might cause a significant challenge in emergency department and an organized approach is needed for successful management and follow-up.

\section{References}

1. Deyo RA (2002) Cascade effects of medical technology. Annu Rev Public Health 23: 23-44. [Crossref]

2. Young WF Jr (2007) Clinical practice. The incidentally discovered adrenal mass. $N$ Engl J Med 356: 601-610. [Crossref]

3. Beigelman-Aubry C, Hill C, Grenier PA (2007). Management of an incidentally discovered pulmonary nodule. Eur Radiol 17: 449-466. [Crossref]

4. Mold JW, Stein HF (1986) The cascade effect in the clinical care of patients. $N$ Engl $J$ Med 314: 512-514. [Crossref]

5. Badiger S, Akkasaligar PT (2014) Medical imaging techniques in clinical medicine. Int J Med Pharm Sci Eng 8: 812-817.

6. Hiremath PS, Akkasaligar PT, Badiger S (2010) Speckle reducing contourlet transform for medical ultrasound images. Int J Comput Inform Eng 4: 284-291.

7. Badiger S, Akkasaligar PT (2012) Electrocardiography-pericarditis, pericardial effusion and cardiac tamponade. Int J Intern Med 1: 37-41

8. Badiger S (2012) Echocardiography in pericardial effusion. Int J Curr Res 4: 100-102.

9. Hiremath PS, Akkasaligar PT, Badiger S (2011) Performance comparison of wavelet transform and contourlet transform based methods for despeckling medical ultrasound images. Int J Comput Applic 26: 34-41.

10. Barboza R, Fox JH, Shaffer LE, Opalek JM, Farooki S (2009) Incidental findings in the cervical spine at CT for trauma evaluation. AJR Am J Roentgenol 192: 725-729. [Crossref]

11. Thompson RJ, Wojcik SM, Grant WD, Ko PY (2011) Incidental Findings on CT Scans in the Emergency Department. Emerg Med Int 2011: 624847. [Crossref]

12. Green DE, Woodward PJ (2005) The management of indeterminate incidental findings detected at abdominal CT. Semin Ultrasound CT MR 26: 2-13. [Crossref]

13. Paluska TR, Sise MJ, Sack DI, Sise CB, Egan MC, et al. (2007) Incidental CT findings in trauma patients: incidence and implications for care of the injured. J Trauma 62 : 157-161. [Crossref]

14. Ekeh AP, Walusimbi M, Brigham E, Woods RJ, McCarthy MC (2010) The prevalence of incidental findings on abdominal computed tomography scans of trauma patients. $J$ Emerg Med 38: 484-489. [Crossref]

15. Devine AS, Jackson CS, Lyons L, Mason JD (2010) Frequency of incidental findings on computed tomography of Trauma patients. West J Emerg Med 11: 24-27. [Crossref]

16. Munk MD, Peitzman AB, Hostler DP, Wolfson AB (2010) Frequency and follow- up of incidental findings on Trauma computed tomography scans: Experience at level one Trauma center. J Emerg Med 38: 346-350. [Crossref]

17. Bordignon KC, Arruda WO (2002) CT scan findings in mild head trauma: a series of 2,000 patients. Arq Neuropsiquiatr 60: 204-210. [Crossref]

18. Eskandary H, Sabba M, Khajehpour F, Eskandari M (2005) Incidental findings in brain computed tomography scans of 3000 head trauma patients. Surg Neurol 63: 550-553. [Crossref]

19. Morteza ST, Hemadi H, Sajadi NM, Jalali AH, Eftekharpour D (2010) Evaluation of Incidental Findings in Brain CT Scans of Mild Head Trauma Patients (GCS: Thirteen to Fifteen). Iran J Cancer Prev 3: 32-35.

Copyright: (C2019 Badiger S. This is an open-access article distributed under the terms of the Creative Commons Attribution License, which permits unrestricted use, distribution, and reproduction in any medium, provided the original author and source are credited. 